\title{
Inequalities for Single Crystal Ribbon Growth by Edge-Defined Film-Fed Growth Technique
}

\author{
Stefan Balint ${ }^{1}$ and Agneta M. Balint ${ }^{2}$ \\ ${ }^{1}$ Computer Science Department, West University of Timisoara, Bulv. V.Parvan 4, \\ 300223 Timisoara, Romania \\ ${ }^{2}$ Faculty of Physics, West University of Timisoara, Bulv. V.Parvan 4, 300223 Timisoara, Romania \\ Correspondence should be addressed to Agneta M. Balint, balint@physics.uvt.ro
}

Received 24 July 2007; Revised 26 November 2007; Accepted 16 February 2008

Recommended by Kok Teo

\begin{abstract}
A second-order nonlinear differential equation, of which some solutions describe the static meniscus free surface (the static liquid bridge free surface between the shaper and the crystal surface) occurring in single crystal ribbon growth, is analyzed. The analysis is focusing on the dependence of the solutions of the equation on the pressure difference $p$ across the free surface. Inequalities are deduced for $p$, which are necessary or sufficient conditions for the stable and convex free surface of a static meniscus. The obtained results are numerically illustrated in the case of a silicon single crystal ribbon growth. The advantage of these kinds of inequalities is that from them special results can be gleaned concerning the experiment planning and technology design. With this aim this study was undertaken.
\end{abstract}

Copyright (c) 2008 S. Balint and A. M. Balint. This is an open access article distributed under the Creative Commons Attribution License, which permits unrestricted use, distribution, and reproduction in any medium, provided the original work is properly cited.

\section{Introduction}

According to [1], modern engineering needs crystals with prescribed shapes and sizes (ribbon-, rod-, and tube-shaped crystals) that allow one to use them as final products without additional machining. Therefore, crystals of specified sizes and shapes are required to be grown from the melt. This problem appears to be solved by profiled-container crystallization, as in the case of casting. However, this solution is not always acceptable. Container material needs to satisfy a certain set of requirements: they should neither react with the melt nor be wetted by it, they should be high-temperature and aggressive-medium resistant, and so forth. Even if all these requirements are satisfied, perfect-crystal growth is not assured and growing thin plate-shaped crystals from the melt exclude container application completely. There are several growth techniques from the melt which allow the shaping of the lateral crystal surface without contact with the container walls, Czochralski technique, Stepanov technique, edge-defined film-fed growth (EFG) technique, and various modifications thereof. Absence of contact between the 
crystallizing substance and the crucible walls in these techniques enables improvement of crystal structures and a decrease in mechanical stress levels. For these techniques the shapes and the dimensions of the crystals grown are controlled by the interface meniscus-shaping capillary forces and by heat- and mass-exchange conditions in the melt-crystal system. Absence of rigid shaping leads to the advantage mentioned above but causes a number of problems. As the crystal is not restricted by the crucible walls, its cross-section depends upon the growing parameters. Any deviation of the growth rate, temperature conditions results in crystal crosssection changes.

A brief description of the shaped crystal growth process by EFG technique is the following. A shaper in which a capillary channel is manufactured is introduced in the melt. Through the capillary channel, the melt moves from the crucible to the upper surface of the shaper where a thin film is formed. The boundary of this film is attached to the edge of the shaper. When a small piece of crystal (called the seed hereafter) is immersed into this film, an equilibrium melt column embracing the surface of the seed immersed is formed. The column formation and catching are caused by the capillary forces present. Such a liquid configuration is usually called a meniscus. Let the temperature of the meniscus upper horizontal section be equal to the temperature of melt crystallization, that is, above the plane of this section the melt transforms into a solid phase. Now, we set the liquid phase into upward motion with the constant rate, $v$, keeping the position of the phase-transition plane invariable by selection of the heat conditions. When the motion starts, the crystallized portion of the meniscus will continuously form a solid upward or downward tapering body. In the particular case when the line tangent to the liquid meniscus free surface at the phase-transition level makes a specific angle $\alpha_{g}\left(\alpha_{g}=\right.$ angle of growth $=$ material constant $)$ with the vertical, and the tangent to the liquid meniscus free surface at the edge of the shaper makes a specific angle $\alpha_{c}\left(\alpha_{c}=\right.$ contact angle $=$ material constant $)$ with the horizontal, the crystallized portion of the meniscus will have constant cross-section equal to the meniscus-section at the crystallization plane level.

Therefore, to grow a crystal with specific shape and cross-section by EFG technique, a corresponding shaper design should be selected and it should be ensured that the meniscus free surface satisfies the above conditions concerning the growth angle $\alpha_{g}$ and the contact angle $\alpha_{c}$ during the pulling process.

For a single crystal ribbon, rod and tube growth by the edge-defined film-fed growth (EFG) technique, in hydrostatic approximation the free surface of the static meniscus is described by the Laplace capillary equation $[1,2]$ :

$$
\gamma \cdot\left(\frac{1}{R_{1}}+\frac{1}{R_{2}}\right)+\rho \cdot g \cdot z=p
$$

Here $\gamma$ is the melt surface tension, $\rho$ denotes the melt density, $g$ is the gravitational acceleration, $R_{1}, R_{2}$ denote the main radii of the free surface curvature at a point $M$ of the free surface, $z$ is the coordinate of $M$ with respect to the $O z$ axis, directed vertically upwards, and $p$ is the pressure difference across the surface $p=p_{m}-p_{g}-\rho \cdot g \cdot H$. Here $p_{m}$ denotes the pressure in the meniscus melt, $p_{g}$ denotes the pressure in the gas, and $H$ denotes the melt column height between the horizontal crucible melt level and the shaper top level (see Figure 1).

To calculate the meniscus surface shape, it is convenient to employ the Laplace equation, represented in (1.1), in its differential form. This form of (1.1) can be obtained as a necessary condition for the minimum of the free energy of the melt column. 


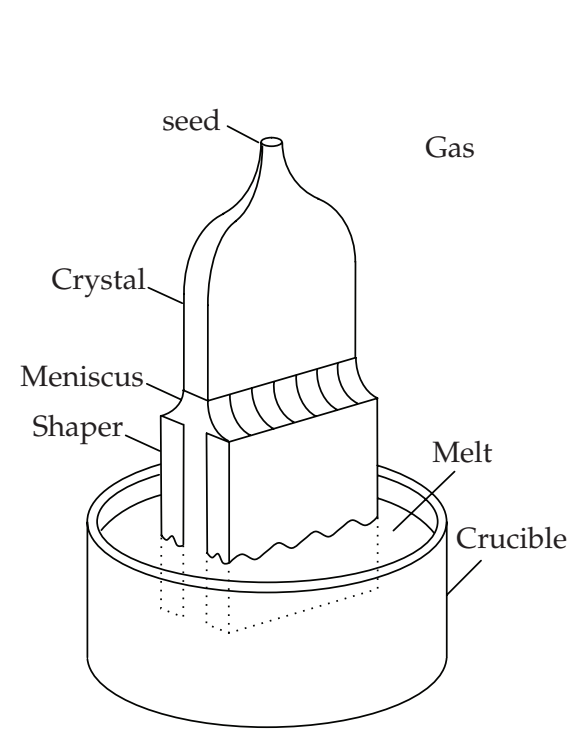

(a)

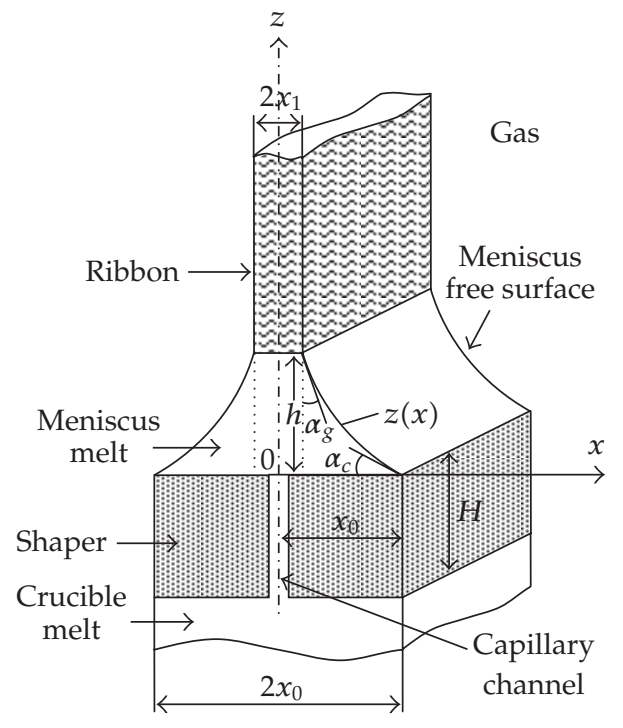

(b)

Figure 1: Meniscus geometry in the ribbon growth by EFG method. (3D representation.)

For the growth of a single crystal ribbon of half thickness $x_{1}, 0<x_{1}<x_{0}$, the differential equation for plan symmetric meniscus surface is given by the formula [1]:

$$
z^{\prime \prime}=\frac{\rho \cdot g \cdot z-p}{\gamma}\left[1+\left(z^{\prime}\right)^{2}\right]^{3 / 2}, \text { for } 0<x_{1} \leq x \leq x_{0}
$$

which is the Euler equation for the free energy functional [1]

$$
I(z)=\int_{x_{1}}^{x_{0}}\left\{\gamma \cdot\left[1+\left(z^{\prime}\right)^{2}\right]^{1 / 2}+\frac{1}{2} \cdot \rho \cdot g \cdot z^{2}-p \cdot z\right\} d x, \quad z\left(x_{1}\right)=h>0, z\left(x_{0}\right)=0 .
$$

Up until 1993-1994, knowledge concerning the dependence of the shape of the meniscus free surface on the pressure difference $p$ across the free surface for small and large Bond numbers in the case of the growth of single crystal rods by the EFG technique is summarized in [1]. According to [1], for the general differential equation describing the free surface of the meniscus, there is no complete analysis and solution. For the general equation, only numerical integrations were carried out for a number of process parameter values that were of practical interest at the time.

In [3], the authors investigate the pressure influence on the meniscus shape for rods, in the case of middle-range Bond numbers (i.e., $B_{0}=1$ ) which most frequently occurs in practice and has been left out of the regular study in [1]. They use a numerical approach in this case to solve the meniscus surface equation written in terms of the arc length of the curve. The stability of the static meniscus free surface is analyzed by means of the Jacobi equation. The result is that a large number of static menisci having drop-like shapes are unstable.

The authors of papers [4,5] consider automated crystal-growth processes based on weight sensors and computers. They give an expression for the weight of the meniscus, in contact with crystal and shaper of arbitrary shape, in which there are terms related to the hydrodynamic factor. 
In [6], Rosolenko shows that the hydrodynamic factor is too small to be considered in the automated crystal-growth; and it is not clear which equation (of non-Laplace type) was considered in $[4,5]$ for the meniscus surface.

In [7], the dependence of the static meniscus free surface shape on the pressure change $p$ is analyzed using an approximate meniscus shape for ribbons and $p \geq 0$.

In this paper, the meniscus shape, described by (1.2), is analyzed as a function of $p$ and its static stability is investigated.

\section{Meniscus shape analysis in the case of ribbon growth}

Consider the differential equation

$$
z^{\prime \prime}=\frac{\rho \cdot g \cdot z-p}{r}\left[1+\left(z^{\prime}\right)^{2}\right]^{3 / 2}, \quad \text { for } x \in\left[0, x_{0}\right], x_{0}>0
$$

and $\alpha_{c}, \alpha_{g}$, such that $0<\alpha_{c}<\pi / 2-\alpha_{g}, \alpha_{g} \in(0, \pi / 2)$.

Here $\alpha_{c}$ represents the contact angle between the meniscus free surface and the shaper; and $\alpha_{g}$ represents the growth angle of the single crystal ribbon. Both are material constants.

The conditions $\alpha_{c}<\pi / 2-\alpha_{g}$ and $\alpha_{g} \in(0, \pi / 2)$ are verified in the growth of classical semiconductor materials, when the shaper is not wetted by the melt.

Definition 2.1. A solution $z=z(x)$ of (2.1) describes the free surface of a static meniscus on the interval $\left[x_{1}, x_{0}\right]\left(0<x_{1}<x_{0}\right)$ if it possesses the following properties:

(i) $z^{\prime}\left(x_{1}\right)=-\tan \left(\pi / 2-\alpha_{g}\right)$,

(ii) $z^{\prime}\left(x_{0}\right)=-\tan \alpha_{c}$,

(iii) $z\left(x_{0}\right)=0$ and $z(x)$ is strictly decreasing on $\left[x_{1}, x_{0}\right]$.

The described free surface is convex on $\left[x_{1}, x_{0}\right]$ if in addition the following inequality holds:

$$
\text { (iv) } z^{\prime \prime}(x)>0 \text { for all } x \in\left[x_{1}, x_{0}\right] \text {. }
$$

\section{Comment}

Conditions (i) and (ii) insure that at the left and right ends of the meniscus, the necessary conditions for obtaining a ribbon with constant cross-section are verified. Condition (iii) insures that the right end of the meniscus is attached to the edge of the shaper and corresponds to the situation when the temperature decreases if $z$ increases.

Theorem 2.2. If there exists a solution $z(x)$ of (2.1) which describes the convex free surface of a static meniscus on the closed interval $\left[x_{1}, x_{0}\right]$, then the following inequalities hold:

$$
-\gamma \cdot \frac{\pi / 2-\left(\alpha_{c}+\alpha_{g}\right)}{x_{0}-x_{1}} \cdot \cos \alpha_{c} \leq p \leq-\gamma \cdot \frac{\pi / 2-\left(\alpha_{c}+\alpha_{g}\right)}{x_{0}-x_{1}} \cdot \sin \alpha_{g}+\rho \cdot g \cdot\left[x_{0}-x_{1}\right] \cdot \tan \left(\frac{\pi}{2}-\alpha_{g}\right) .
$$


Proof. Let $z(x)$ be a solution of (2.1), which describes the convex free surface of a static meniscus on the closed interval $\left[x_{1}, x_{0}\right]$ and $\alpha(x)=-\arctan z^{\prime}(x)$. The function $\alpha(x)$ verifies

$$
\alpha^{\prime}(x)=\frac{p-\rho \cdot g \cdot z(x)}{\gamma} \cdot \frac{1}{\cos \alpha(x)}
$$

and the boundary conditions

$$
\alpha\left(x_{1}\right)=\frac{\pi}{2}-\alpha_{g}, \quad \alpha\left(x_{0}\right)=\alpha_{c} .
$$

Hence, by the mean value theorem, there exists $x^{\prime} \in\left(x_{1}, x_{0}\right)$ such that the following equality holds:

$$
p=-\gamma \cdot \frac{\pi / 2-\left(\alpha_{c}+\alpha_{g}\right)}{x_{0}-x_{1}} \cdot \cos \alpha\left(x^{\prime}\right)+\rho \cdot g \cdot z\left(x^{\prime}\right) .
$$

On the other hand, from $z^{\prime \prime}(x)>0$ for all $x \in\left[x_{1}, x_{0}\right]$, it follows that $z^{\prime}(x)$ is strictly increasing and $\alpha(x)=-\arctan z^{\prime}(x)$ is strictly decreasing on $\left[x_{1}, x_{0}\right]$. Therefore, on the closed interval $\left[x_{1}, x_{0}\right]$ the following inequalities hold:

$$
\begin{gathered}
\alpha_{c} \leq \alpha(x) \leq \frac{\pi}{2}-\alpha_{g} \\
-\tan \left(\frac{\pi}{2}-\alpha_{g}\right) \leq z^{\prime}(x) \leq-\tan \alpha_{c} .
\end{gathered}
$$

Inequalities (2.6) imply

$$
\begin{aligned}
-\gamma \cdot \frac{\pi / 2-\left(\alpha_{c}+\alpha_{g}\right)}{x_{0}-x_{1}} \cdot \cos \alpha_{c} & \leq-\gamma \cdot \frac{\pi / 2-\left(\alpha_{c}+\alpha_{g}\right)}{x_{0}-x_{1}} \cdot \cos \alpha\left(x^{\prime}\right) \\
& \leq-\gamma \cdot \frac{\pi / 2-\left(\alpha_{c}+\alpha_{g}\right)}{x_{0}-x_{1}} \cdot \sin \alpha_{g},
\end{aligned}
$$

and the inequalities (2.7), by integration on the closed interval $\left[x^{\prime}, x_{0}\right]$, imply

$$
\rho \cdot g \cdot\left(x_{0}-x^{\prime}\right) \cdot \tan \alpha_{c} \leq \rho \cdot g \cdot z\left(x^{\prime}\right) \leq \rho \cdot g \cdot\left[x_{0}-x^{\prime}\right] \cdot \tan \left(\frac{\pi}{2}-\alpha_{g}\right) .
$$

From (2.5), (2.8), and (2.9) it follows that for $p$ the inequalities (2.2) hold.

\section{Consequences}

If $x_{1}=x_{0} / n$, with $n>1$, then the inequalities (2.2) can be written as

$$
\begin{aligned}
& -n \cdot \gamma \cdot \frac{\pi / 2-\left(\alpha_{c}+\alpha_{g}\right)}{(n-1) \cdot x_{0}} \cdot \cos \alpha_{c} \\
& \quad \leq p \leq-n \cdot \gamma \cdot \frac{\pi / 2-\left(\alpha_{c}+\alpha_{g}\right)}{(n-1) \cdot x_{0}} \cdot \sin \alpha_{g}+\frac{\rho \cdot g \cdot x_{0} \cdot(n-1)}{n} \cdot \tan \left(\frac{\pi}{2}-\alpha_{g}\right) . \\
& \text { If } n \rightarrow+\infty \text {, then } x_{1} \rightarrow 0 \text { and }(2.10) \text { becomes } \\
& -\gamma \cdot \frac{\pi / 2-\left(\alpha_{c}+\alpha_{g}\right)}{x_{0}} \cdot \cos \alpha_{c} \leq p \leq-\gamma \cdot \frac{\pi / 2-\left(\alpha_{c}+\alpha_{g}\right)}{x_{0}} \cdot \sin \alpha_{g}+\rho \cdot g \cdot x_{0} \cdot \tan \left(\frac{\pi}{2}-\alpha_{g}\right) . \\
& \text { If } n \rightarrow 1 \text {, then } x_{1} \rightarrow x_{0} \text { and } p \rightarrow-\infty .
\end{aligned}
$$


Theorem 2.3. Let $n>1$. If $p$ satisfies

$$
p<-n \cdot \gamma \cdot \frac{\pi / 2-\left(\alpha_{c}+\alpha_{g}\right)}{(n-1) \cdot x_{0}} \cdot \cos \alpha_{c}
$$

then there exists $x_{1}, x_{1} \in\left[x_{0} / n, x_{0}\right]$ such that the solution $z(x)$ of the initial value problem

$$
\begin{gathered}
z^{\prime \prime}=\frac{\rho \cdot g \cdot z-p}{\gamma} \cdot\left[1+\left(z^{\prime}\right)^{2}\right]^{3 / 2}, \\
z\left(x_{0}\right)=0, \quad z^{\prime}\left(x_{0}\right)=-\tan \alpha_{c}, \quad 0<\alpha_{c}<\frac{\pi}{2}-\alpha_{g}, \alpha_{g} \in\left(0, \frac{\pi}{2}\right),
\end{gathered}
$$

on the interval $\left[x_{1}, x_{0}\right]$ describes the convex free surface of a static meniscus.

Proof. Let us consider the maximal interval $I$ on which the solution of the initial value problem (2.13) exists.

Since $p<0$ we have

$$
z^{\prime \prime}\left(x_{0}\right)>0, \quad z^{\prime}\left(x_{0}\right)<0, \quad z^{\prime}\left(x_{0}\right)>-\tan \left(\frac{\pi}{2}-\alpha_{g}\right)
$$

and there exists $x^{\prime} \in I, x^{\prime}<x_{0}$ such that for any $x \in\left[x^{\prime}, x_{0}\right]$ the following inequalities hold:

$$
z^{\prime \prime}(x)>0, \quad z^{\prime}(x)<0, \quad z^{\prime}(x)>-\tan \left(\frac{\pi}{2}-\alpha_{g}\right) .
$$

Let $x^{*}$ be now defined by

$$
x^{*}=\inf \left\{x^{\prime} \in I \mid x^{\prime}<x_{0} \text { such that for any } x \in\left[x^{\prime}, x_{0}\right] \text { inequalities (2.15) hold }\right\} \text {. }
$$

Now we show that $x^{*}$ is finite, that is, $x^{*}>-\infty$. For this purpose, consider an arbitrary $x^{\prime}$ such that $x^{*}<x^{\prime}<x_{0}$. For every $x$ such that $x \in\left[x^{\prime}, x_{0}\right]$ we have $-\tan \left(\pi / 2-\alpha_{g}\right)<z^{\prime}(x) \leq-\tan \alpha_{c}$ and hence,

$$
0 \leq\left[x_{0}-x\right] \cdot \tan \alpha_{c} \leq z(x) \leq\left[x_{0}-x\right] \cdot \tan \left(\frac{\pi}{2}-\alpha_{g}\right)
$$

Therefore, using (2.13), we deduce that $z(x)$ satisfies

$$
z^{\prime \prime}(x) \geq-\frac{p}{r} \text { on the interval }\left[x^{\prime}, x_{0}\right]
$$

Hence,

$$
z^{\prime}(x) \leq \frac{p}{\gamma} \cdot\left[x_{0}-x\right]-\tan \alpha_{c} \text { on the interval }\left[x^{\prime}, x_{0}\right]
$$

and, in particular,

$$
z^{\prime}\left(x^{\prime}\right) \leq \frac{p}{\gamma} \cdot\left[x_{0}-x^{\prime}\right]-\tan \alpha_{c}
$$


Assuming that $x^{*}=-\infty$, we can choose $x^{\prime}$ such that

$$
x^{\prime}<x_{0}-\frac{\gamma}{p} \cdot\left[\tan \alpha_{c}-\tan \left(\frac{\pi}{2}-\alpha_{g}\right)\right]
$$

and obtain in this way that $z^{\prime}\left(x^{\prime}\right)<-\tan \left(\pi / 2-\alpha_{g}\right)$, which is clearly false. It follows in this way that $x^{*}>-\infty$.

Since for $x>x^{*}$ and $x$ tending to $x^{*}, z(x)$ and $z^{\prime}(x)$ have finite limits which satisfy

$$
\begin{gathered}
{\left[x_{0}-x^{*}\right] \cdot \tan \alpha_{c} \leq \lim _{\substack{x \rightarrow x^{*} \\
x>x^{*}}} z(x) \leq\left[x_{0}-x^{*}\right] \cdot \tan \left(\frac{\pi}{2}-\alpha_{g}\right)} \\
-\tan \left(\frac{\pi}{2}-\alpha_{g}\right) \leq \lim _{\substack{x \rightarrow x^{*} \\
x>x^{*}}} z^{\prime}(x) \leq \frac{p}{\gamma} \cdot\left[x_{0}-x^{*}\right]-\tan \alpha_{c} .
\end{gathered}
$$

We can consider $z\left(x^{*}+0\right), z^{\prime}\left(x^{*}+0\right)$ and the initial value problem corresponding to these initial data. The solution of this initial value problem extends the solution of the initial value problem (2.13) and so it follows that $x^{*}$ belongs to $I$.

Considering the values of $z^{\prime \prime}\left(x^{*}\right)$ and $z^{\prime}\left(x^{*}\right)$, it is clear that they have to satisfy the following inequalities:

$$
z^{\prime \prime}\left(x^{*}\right) \geq 0, \quad z^{\prime}\left(x^{*}\right) \leq 0, \quad z^{\prime}\left(x^{*}\right) \geq-\tan \left(\frac{\pi}{2}-\alpha_{g}\right)
$$

Due to the fact that $x^{*}$ is infimum, one of the above inequalities has to be equality.

The equality $z^{\prime \prime}\left(x^{*}\right)=0$ implies that $\rho \cdot g \cdot z\left(x^{*}\right)=p$. This equality is impossible because $p<0$ and $\rho \cdot g \cdot z\left(x^{*}\right)>\rho \cdot g \cdot\left[x_{0}-x^{*}\right] \cdot \tan \alpha_{c}>0$.

The equality $z^{\prime}\left(x^{*}\right)=0$ is impossible because $z^{\prime}\left(x^{*}\right) \leq-\tan \alpha_{c}<0$.

It follows in this way that the following equality holds:

$$
z^{\prime}\left(x^{*}\right)=-\tan \left(\frac{\pi}{2}-\alpha_{g}\right)
$$

In order to show that $x^{*}$ belongs to the interval $\left[x_{0} / n, x_{0}\right]$, we apply the mean value theorem for $\alpha(x)$ defined in the proof of Theorem 2.2 and write the equality

$$
\alpha_{c}-\left(\frac{\pi}{2}-\alpha_{g}\right)=\alpha^{\prime}(\xi) \cdot\left[x_{0}-x^{*}\right]
$$

where,

$$
\alpha^{\prime}(\xi)=\frac{p-\rho \cdot g \cdot z(\xi)}{\gamma} \cdot \frac{1}{\cos \alpha(\xi)}, \quad \xi \in\left(x^{*}, x_{0}\right)
$$


Hence,

$$
\begin{aligned}
x_{0}-x^{*} & =\frac{\alpha_{c}-\left(\pi / 2-\alpha_{g}\right)}{\alpha^{\prime}(\xi)} \\
& =\frac{\alpha_{c}+\alpha_{g}-\pi / 2}{(p-\rho \cdot g \cdot z(\xi)) / \gamma \cdot 1 / \cos \alpha(\xi)} \\
& <\frac{\pi / 2-\left(\alpha_{c}+\alpha_{g}\right)}{-p / \gamma \cdot 1 / \cos \alpha(\xi)} \\
& <\frac{\pi / 2-\left(\alpha_{c}+\alpha_{g}\right)}{n /(n-1) \cdot x_{0} \cdot\left[\pi / 2-\left(\alpha_{c}+\alpha_{g}\right)\right] \cdot \cos \alpha_{c} / \cos \alpha(\xi)} \\
& <\frac{n-1}{n} \cdot x_{0} \\
& =x_{0}-\frac{1}{n} \cdot x_{0} .
\end{aligned}
$$

From (2.27), we obtain

$$
x^{*}>\frac{1}{n} \cdot x_{0}
$$

Inequality (2.28) shows that $x^{*} \in\left[x_{0} / n, x_{0}\right]$. Taking $x_{1}=x^{*}$ we obtain the conclusion of the theorem.

Remark 2.4. If for $n>n^{\prime}>1$ and $p$ the following inequalities hold:

$$
\begin{aligned}
& -n^{\prime} \cdot \gamma \cdot \frac{\pi / 2-\left(\alpha_{c}+\alpha_{g}\right)}{\left(n^{\prime}-1\right) \cdot x_{0}} \cdot \sin \alpha_{g}+\frac{\rho \cdot g \cdot x_{0} \cdot\left(n^{\prime}-1\right)}{n^{\prime}} \cdot \tan \left(\frac{\pi}{2}-\alpha_{g}\right) \\
& \quad \leq p<-n \cdot \gamma \cdot \frac{\pi / 2-\left(\alpha_{c}+\alpha_{g}\right)}{(n-1) \cdot x_{0}} \cdot \cos \alpha_{c \prime}
\end{aligned}
$$

then there exists $x_{1} \in\left[x_{0} / n, x_{0} / n^{\prime}\right]$ such that the solution $z(x)$ of the initial value problem (2.13) on the interval $\left[x_{1}, x_{0}\right]$ describes a convex free surface of a static meniscus.

The existence of $x_{1}$ and the inequality $x_{1} \geq x_{0} / n$ follows from Theorem 2.3. The inequality $x_{1} \leq x_{0} / n^{\prime}$ follows from Theorem 2.2.

Theorem 2.5. If a solution $z_{1}=z_{1}(x)$ of (2.1) describes a convex free surface of a static meniscus on the interval $\left[x_{1}, x_{0}\right]\left(0<x_{1}<x_{0}\right)$, then it is a weak minimum for the total free energy functional of the melt column:

$$
\begin{gathered}
I(z)=\int_{x_{1}}^{x_{0}}\left\{r \cdot\left[1+\left(z^{\prime}\right)^{2}\right]^{1 / 2}+\frac{1}{2} \cdot \rho \cdot g \cdot z^{2}-p \cdot z\right\} \cdot d x \\
z\left(x_{1}\right)=z_{1}\left(x_{1}\right) \\
z\left(x_{0}\right)=0 .
\end{gathered}
$$


Proof. Since (2.1) is the Euler equation for (2.30), it is sufficient to prove that the Legendre and Jacobi conditions are satisfied in this case.

Denote by $F\left(z, z^{\prime}\right)$ the function defined as

$$
F\left(z, z^{\prime}\right)=\gamma \cdot\left[1+\left(z^{\prime}\right)^{2}\right]^{1 / 2}+\frac{1}{2} \cdot \rho \cdot g \cdot z^{2}-p \cdot z
$$

It is easy to see that we have

$$
\frac{\partial^{2} F}{\partial z^{\prime 2}}=\frac{\gamma}{\left[1+\left(z^{\prime}\right)^{2}\right]^{3 / 2}}>0
$$

Hence, the Legendre condition is satisfied.

The Jacobi equation:

$$
\left[\frac{\partial^{2} F}{\partial z^{2}}-\frac{d}{d x}\left(\frac{\partial^{2} F}{\partial z \partial z^{\prime}}\right)\right] \cdot \eta-\frac{d}{d x}\left[\frac{\partial^{2} F}{\partial z^{\prime 2}} \cdot \eta^{\prime}\right]=0
$$

in this case is defined by

$$
\frac{d}{d x}\left(\frac{r}{\left[1+\left(z^{\prime}\right)^{2}\right]^{3 / 2}} \cdot \eta^{\prime}\right)-\rho \cdot g \cdot \eta=0
$$

For (2.34), the following inequalities hold:

$$
\frac{\gamma}{\left[1+\left(z^{\prime}\right)^{2}\right]^{3 / 2}} \geq \gamma \cdot \cos ^{3}\left(\frac{\pi}{2}-\alpha_{g}\right)=\gamma \cdot \sin ^{3} \alpha_{g}, \quad-\rho \cdot g<0
$$

Hence,

$$
\left(\eta^{\prime} \cdot \gamma \cdot \sin ^{3} \alpha_{g}\right)^{\prime}=0 \quad \text { or } \quad \eta^{\prime \prime}=0
$$

is a Sturm-type upper bound for (2.34) [8].

Since every nonzero solution of (2.36) vanishes at most once on the interval $\left[x_{1}, x_{0}\right]$, the solution $\eta(x)$ of the initial value problem

$$
\begin{gathered}
\frac{d}{d x}\left(\frac{\gamma}{\left[1+\left(z^{\prime}\right)^{2}\right]^{3 / 2}} \cdot \eta^{\prime}\right)-\rho \cdot g \cdot \eta=0 \\
\eta\left(x_{1}\right)=0 \\
\eta^{\prime}\left(x_{1}\right)=1
\end{gathered}
$$

has only one zero on the interval $\left[x_{1}, x_{0}\right]$. Hence the Jacobi condition is satisfied.

Definition 2.6. A solution $z=z(x)$ of (2.1) which describes a static meniscus free surface is said to be stable if it is a weak minimum of the free energy functional of the melt column. 
Remark 2.7. Theorem 2.5 shows that if $z=z(x)$ describes the convex free surface of a static meniscus on the interval $\left[x_{1}, x_{0}\right]$, then it is stable.

Theorem 2.8. If the solution $z=z(x)$ of the initial value problem (2.13) is concave (i.e., $z^{\prime \prime}(x)<0$ ) on the interval $\left[x_{1}, x_{0}\right]\left(0<x_{1}<x_{0}\right)$, then it does not describe the free surface of a static meniscus on $\left[x_{1}, x_{0}\right]$.

Proof. $z^{\prime \prime}(x)<0$ on $\left[x_{1}, x_{0}\right]$ implies that $z^{\prime}(x)$ is strictly decreasing on $\left[x_{1}, x_{0}\right]$. Hence, $z^{\prime}\left(x_{1}\right)>$ $z^{\prime}\left(x_{0}\right)=-\tan \alpha_{c}>-\tan \left(\pi / 2-\alpha_{g}\right)$.

Theorem 2.9. If $p$ satisfies the inequality

$$
\rho \cdot g \cdot x_{0} \cdot \tan \alpha_{c}<p
$$

then the solution of the initial value problem (2.13) is concave on the interval $I \cap\left[0, x_{0}\right]$ where $I$ is the maximal interval of the existence of the solution $z=z(x)$.

Proof. Since $z^{\prime \prime}\left(x_{0}\right)<0$, there exists $x^{\prime} \in I \cap\left(-\infty, x_{0}\right]$ such that for any $x \in\left[x^{\prime}, x_{0}\right]$ we have $z^{\prime \prime}(x)<0$. Hence $z^{\prime}(x)$ is strictly decreasing on $\left[x^{\prime}, x_{0}\right]$ and $z^{\prime}(x) \geq-\tan \alpha_{c}$ for every $x \in$ $\left[x^{\prime}, x_{0}\right]$. It follows that $z(x) \leq\left(x_{0}-x\right) \cdot \tan \alpha_{c}$ for every $x \in\left[x^{\prime}, x_{0}\right]$. Let $x^{*}$ now be defined as

$$
x^{*}=\operatorname{Inf}\left\{x^{\prime} \mid x^{\prime} \in I \cap\left(-\infty, x_{0}\right), z^{\prime \prime}(x)<0 \text { on }\left[x^{\prime}, x_{0}\right]\right\} .
$$

We show that $x^{*} \leq 0$. For this purpose, assume the contrary, namely that $x^{*}>0$. Since $\sup \left\{z^{\prime \prime}(x) \mid x \in\left(x^{*}, x_{0}\right]\right\}=0$ for every $\varepsilon>0$ there exists $x^{\prime}$ such that $0<x^{*}<x^{\prime}<x_{0}$ and $-\varepsilon<z^{\prime \prime}\left(x^{\prime}\right)<0$. Hence $\rho \cdot g \cdot z\left(x^{\prime}\right)-p>-\varepsilon / \gamma \cdot\left[1+\left(z^{\prime}\right)^{2}\right]^{-3 / 2}>-\varepsilon / \gamma$ and, due to the inequalities

$$
\rho \cdot g \cdot z\left(x^{\prime}\right)-p \leq \rho \cdot g \cdot\left(x_{0}-x^{\prime}\right) \cdot \tan \alpha_{c}-p \leq \rho \cdot g \cdot\left(x_{0}-x^{*}\right) \cdot \tan \alpha_{c}-p<0,
$$

we obtain that the following inequalities hold:

$$
-\frac{\varepsilon}{\gamma}<-\frac{\varepsilon}{\gamma} \cdot\left[1+\left(z^{\prime}\left(x^{\prime}\right)\right)^{2}\right]^{-3 / 2}<\rho \cdot g \cdot\left(x_{0}-x^{*}\right) \cdot \tan \alpha_{c}-p .
$$

It is clear that $-\varepsilon / \gamma<\rho \cdot g \cdot\left(x_{0}-x^{*}\right) \cdot \tan \alpha_{c}-p<0$ is not true for every $\varepsilon>0$. Therefore, the assumption that $x^{*}>0$ is absurd. It follows that $x^{*} \leq 0$, which proves the theorem.

\section{Consequence}

If $p$ verifies the inequality (2.38), then there does not exist $x_{1} \in\left[0, x_{0}\right)$ such that the solution of the initial value problem (2.13) describes the free surface of a static meniscus on $\left[x_{1}, x_{0}\right]$.

\section{Numerical illustration}

Numerical computations were performed for Si ribbon using the following numerical data [7]:

$$
\begin{gathered}
x_{0}=3 \cdot 10^{-4}[\mathrm{~m}] ; \quad x_{1}=10^{-4}[\mathrm{~m}] ; \quad n=3 ; \quad \alpha_{c}=0.523[\mathrm{rad}] ; \quad \alpha_{g}=0.192[\mathrm{rad}] ; \\
\rho=25 \cdot 10^{2}\left[\mathrm{~kg} / \mathrm{m}^{3}\right] ; \quad \gamma=720 \cdot 10^{-3}[\mathrm{~N} / \mathrm{m}] ; \quad g=9.81\left[\mathrm{~m} / \mathrm{s}^{2}\right]
\end{gathered}
$$

and the software MathCAD Professional. 


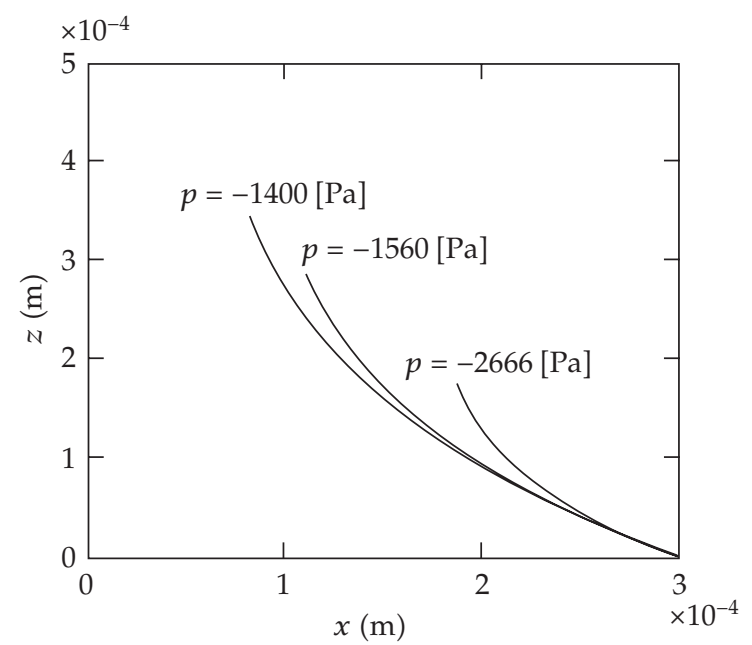

Figure 2: $z$ versus $x$ for $p=-2666 ;-1560 ;-1400[\mathrm{~Pa}]$.

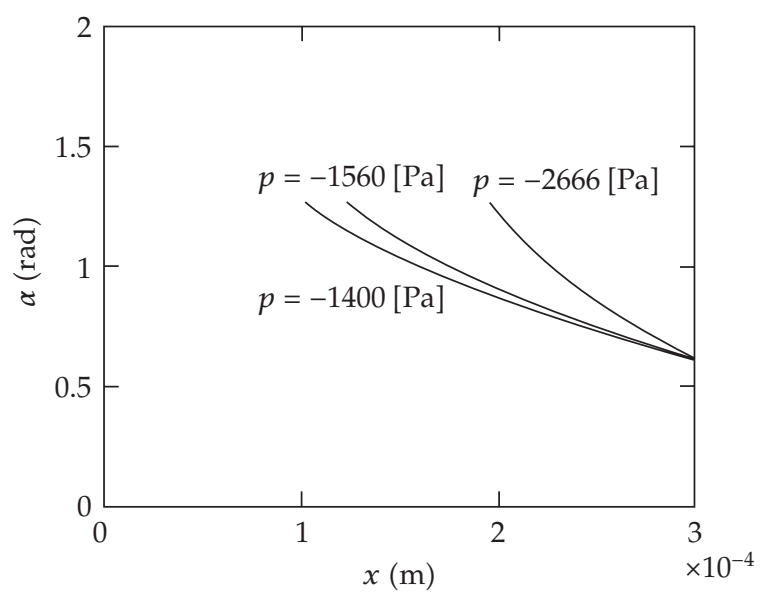

Figure 3: $\alpha$ versus $x$ for $p=-2666 ;-1560 ;-1400[\mathrm{~Pa}]$.

The objective is to illustrate numerically the inequalities obtained in the theoretical part and to see if the necessary conditions are also sufficient conditions or if the sufficient conditions are also necessary. Moreover, the above data are realistic $[7,8]$ and the computed results can be tested against those obtained experimentally in order to evaluate the accuracy of the theoretical predictions. This comparison is not the subject of this paper.

Inequality (2.2) is a necessary condition for the existence of the convex free surface of a static meniscus on the closed interval $\left[x_{1}, x_{0}\right]\left(x_{1}>0\right)$. Is this condition also sufficient? Computation shows that for the considered numerical data, the inequality (2.2) becomes $-2666.4[\mathrm{~Pa}] \leq p \leq-562.2[\mathrm{~Pa}]$.

Numerical integration of the initial value problem (2.13) shows that for $p=-2666 ;-1560$ [Pa] there exists $x^{\prime} \in\left(x_{1}, x_{0}\right)$ such that the solution of the initial value problem is the convex free surface of a static meniscus on $\left[x^{\prime}, x_{0}\right]$, but for $p=-1400[\mathrm{~Pa}]$ there is no $x^{\prime} \in\left[x_{1}, x_{0}\right]$ such that the solution of the initial value problem is a convex meniscus on $\left[x^{\prime}, x_{0}\right]$ (Figures 2 and 3 ).

Consequently, condition (2.2) is a necessary one, but it is not sufficient. 


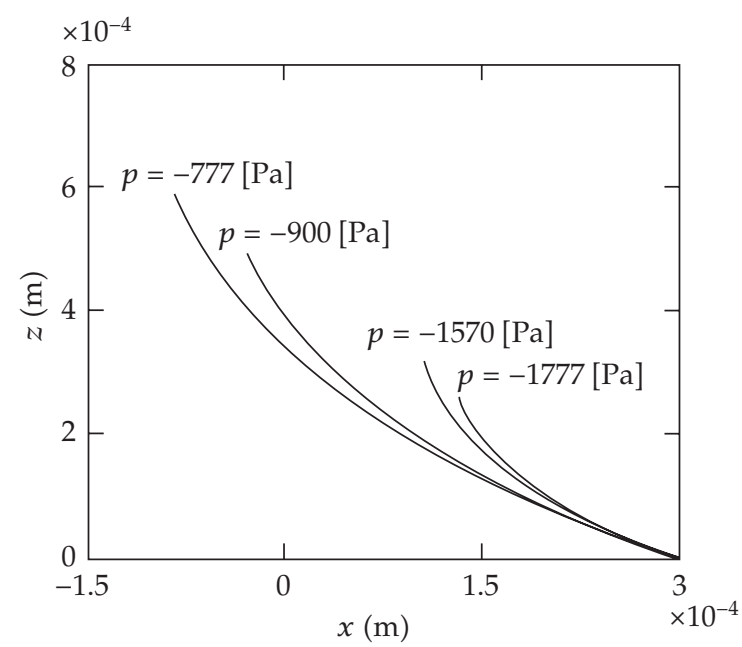

Figure 4: $z$ versus $x$ for $p=-1777 ;-1570 ;-900 ;-777[\mathrm{~Pa}]$.

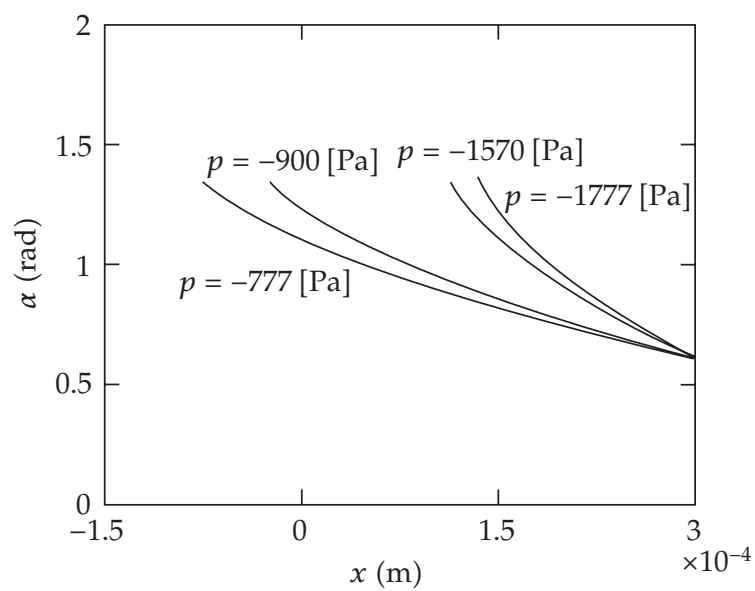

Figure 5: $\alpha$ versus $x$ for $p=-1777 ;-1570 ;-900 ;-777[\mathrm{~Pa}]$.

Inequality (2.11) is a necessary condition for the existence of a point $x^{\prime} \in\left[0, x_{0}\right]$ such that the solution of the initial value problem (2.13) is a convex free surface of a static meniscus on the closed interval $\left[x^{\prime}, x_{0}\right]$. Is this condition also sufficient? Computation made using the same numerical data shows that in this case inequality (2.11) becomes $-1777[\mathrm{~Pa}] \leq p \leq$ $-353.810[\mathrm{~Pa}]$.

Numerical integration of the initial value problem (2.13) shows that for $p=-1777 ;-1570$ $[\mathrm{Pa}]$ there exists $x^{\prime} \in\left(0, x_{0}\right)$ such that the solution of the initial value problem is a convex free surface of a static meniscus on $\left[x^{\prime}, x_{0}\right]$; but for $p=-900 ;-777[\mathrm{~Pa}]$ there is no $x^{\prime} \in\left(0, x_{0}\right)$ such that the solution of the initial value problem (2.13) is a convex free surface of a static meniscus on $\left[x^{\prime}, x_{0}\right]$ (Figures 4 and 5 ).

Consequently, the inequality (2.11) is not a sufficient condition.

Inequality (2.12) is a sufficient condition for the existence of a point $x^{\prime}$ in the interval $\left[x_{0} / n, x_{0}\right]$ such that the solution of the initial value problem (2.13) is a convex free surface of a static meniscus on the interval $\left[x^{\prime}, x_{0}\right]$. Is this condition also a necessary condition? 


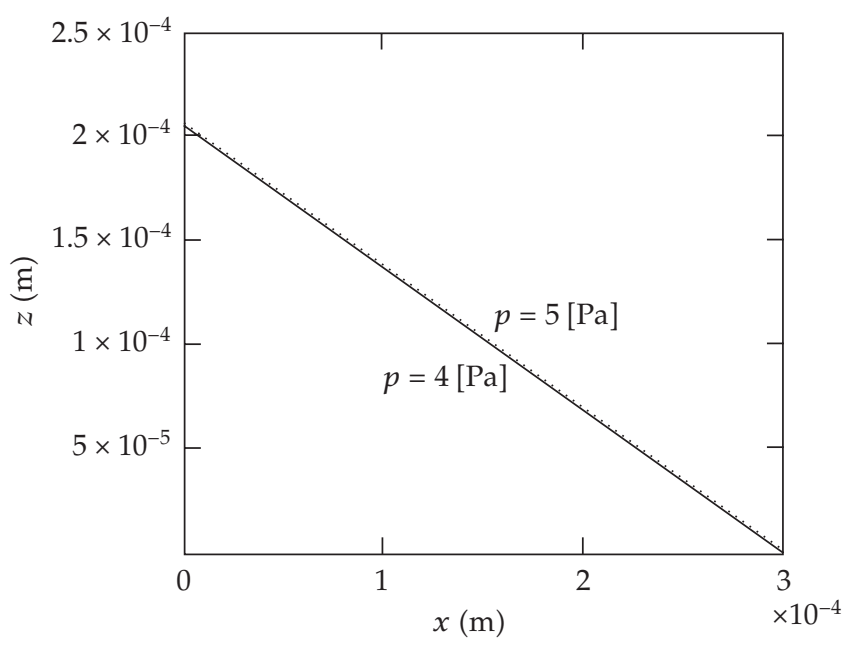

Figure 6: $z$ versus $x$ for $p=4 ; 5[\mathrm{~Pa}]$.

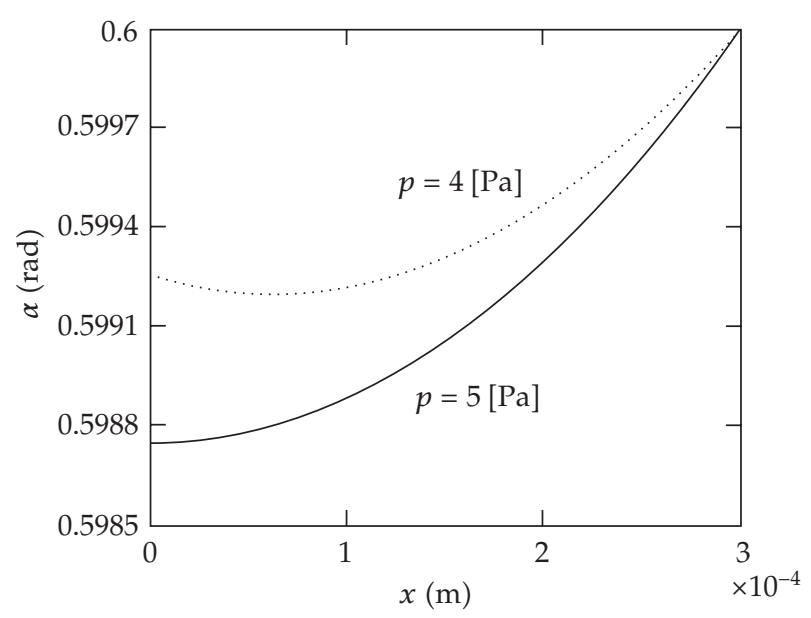

Figure 7: $\alpha$ versus $x$ for $p=4 ; 5[\mathrm{~Pa}]$.

Computation made using the same numerical data shows that in this case $(n=$ 3) inequality (2.12) becomes $p<-2666.3[\mathrm{~Pa}]$. We have already obtained, by numerical integration, that for $p=-1.777 \cdot 10^{3}[\mathrm{~Pa}]$, there exists $x^{\prime} \in\left[x_{0} / 3, x_{0}\right]$ such that the solution of the initial value problem (2.13) is a convex free surface of a static meniscus on the interval $\left[x^{\prime}, x_{0}\right]$. Consequently, the inequality $(2.12)$ is not a necessary condition.

Inequality (2.38) is a sufficient condition which assures that the solution of the initial value problem $(2.13)$ is concave on $\left[0, x_{0}\right]$. Is this condition also a necessary condition?

Computation made using the same numerical data shows that inequality (2.38) becomes $p>4.248[\mathrm{~Pa}]$. Numerical integration of the initial value problem (2.13) shows that for $p=$ $4[\mathrm{~Pa}]$ the solution of the initial value problem (2.13) is convex at the left-hand side and concave at the right-hand side on $\left[0, x_{0}\right]$; and for $p=5[\mathrm{~Pa}]$ the solution of the initial value problem (2.13) is concave on $\left[0, x_{0}\right]$ (Figures 6 and 7 ).

Consequently, in this particular case from practical point of view inequality (2.38) is also a necessary condition. 


\section{Conclusions}

(1) The formulas, which were established for a general configuration and an arbitrary material, are useful in the experiment design by predicting the values of the pressures $p$ which have to be or can be used in order to obtain

(i) stable static menisci corresponding to a given contact angle $\alpha_{c}$, growth angle $\alpha_{g}$, and ribbon half thickness $x_{1}$ (formula (2.2) Theorem 2.2 and formulas (2.10); (2.11)),

(ii) stable static menisci corresponding to a given contact angle $\alpha_{c}$ and growth angle $\alpha_{g}$ on which the growth angle is realized in a prescribed region (formula (2.12) Theorem 2.3 and formula (2.29), and

(iii) globally concave surfaces on which the growth angle $\alpha_{g}$ is not realized.

(2) The numerical illustrations show that the obtained necessary conditions in general are not sufficient conditions and the obtained sufficient conditions in general are not necessary conditions.

\section{Acknowledgments}

The authors would like to thank the anonymous referees for their valuable comments and suggestions which led to an improvement of the manuscript. The authors thank also the Romanian National Authority for Research for supporting the research under the Grant no. $7 / 2007$.

\section{References}

[1] V. A. Tatarchenko, Shaped Crystal Growth, Kluwer Academic Publishers, Dordrecht, The Netherlands, 1993.

[2] R. Finn, Equilibrium Capillary Surfaces, vol. 284 of Grundlehren der Mathematischen Wissenschaften, Springer, New York, NY, USA, 1986.

[3] V. A. Tatarchenko, V. S. Uspenski, E. V. Tatarchenko, J. Ph. Nabot, T. Duffar, and B. Roux, "Theoretical model of crystal growth shaping process," Journal of Crystal Growth, vol. 180, no. 3-4, pp. 615-626, 1997.

[4] A. V. Borodin, V. A. Borodin, V. V. Sidorov, and I. S. Pet'kov, "Influence of growth process parameters on weight sensor readings in the Stepanov (EFG) technique," Journal of Crystal Growth, vol. 198-199, part 1, pp. 215-219, 1999.

[5] A. V. Borodin, V. A. Borodin, and A. V. Zhdanov, "Simulation of the pressure distribution in the melt for sapphire ribbon growth by the Stepanov (EFG) technique," Journal of Crystal Growth, vol. 198-199, part 1, pp. 220-226, 1999.

[6] S. N. Rosolenko, "Menisci masses and weights in Stepanov (EFG) technique: ribbon, rod, tube," Journal of Crystal Growth, vol. 231, no. 1-2, pp. 306-315, 2001.

[7] St. Balint, L. Braescu, A. M. Balint, and R. Szabo, "Modelling the effects of the pressure changes in the case of the growth of a thin sheet in an edge-defined film-fed growth (EFG) System," Journal of Crystal Growth, vol. 283, no. 1-2, pp. 15-30, 2005.

[8] P. Hartman, Ordinary Differential Equations, John Wiley \& Sons, New York, NY, USA, 1964. 